\title{
Universiteit
}

Leiden

The Netherlands

\section{Dynamic ordering and frustration of confined vortex rows studied by mode-locking experiments}

Kokubo, N.; Besseling, R.; Kes, P.H.

\section{Citation}

Kokubo, N., Besseling, R., \& Kes, P. H. (2004). Dynamic ordering and frustration of confined vortex rows studied by mode-locking experiments. Physical Review B, 69(6), 064504. doi:10.1103/PhysRevB.69.064504

Version: $\quad$ Not Applicable (or Unknown)

License: $\quad$ Leiden University Non-exclusive license

Downloaded from: https://hdl.handle.net/1887/77094

Note: To cite this publication please use the final published version (if applicable). 


\title{
Dynamic ordering and frustration of confined vortex rows studied by mode-locking experiments
}

\author{
N. Kokubo, R. Besseling, and P. H. Kes \\ Kamerlingh Onnes Laboratorium, Leiden University, P.O. Box 9504, 2300 RA Leiden, The Netherlands
}

(Received 25 August 2003; published 19 February 2004)

\begin{abstract}
The flow properties of confined vortex matter driven through disordered mesoscopic channels are investigated by mode locking (ML) experiments. The observed ML effects allow us to trace the evolution of both the structure and the number of confined rows and their match to the channel width as function of magnetic field. From a detailed analysis of the ML behavior for the case of three rows we obtain (i) the pinning frequency $f_{p}$, (ii) the onset frequency $f_{c}$ for ML ( $\propto$ ordering velocity), and (iii) the fraction $L_{\mathrm{ML}} / L$ of coherently moving three-row regions in the channel. The field dependence of these quantities shows that, at matching, where $L_{\mathrm{ML}}$ is maximum, the pinning strength is small and the ordering velocity is low, while at mismatch, where $L_{\mathrm{ML}}$ is small, both the pinning force and the ordering velocity are enhanced. Further, we find that $f_{c} \propto f_{p}^{2}$, consistent with the dynamic ordering theory of Koshelev and Vinokur. The microscopic nature of the flow and the ordering phenomena will also be discussed.
\end{abstract}

DOI: 10.1103/PhysRevB.69.064504

PACS number(s): 74.25.Qt, 83.50.Ha, 74.78.Na

\section{INTRODUCTION}

Vortex arrays (VA's) in type-II superconductors are exemplary systems to study nonequilibrium states of driven periodic media in various pinning environments. A particularly interesting phenomenon in this context is that of a dynamic transition from an elastic, coherent flow state at large velocities to a plastic, incoherent flow state at small velocities. The first theoretical description of this issue was provided by Koshelev and Vinokur (KV) (Ref. 1) and refined in subsequent studies ${ }^{2-5}$ which predicted various novel flow states, including a moving glass characterized by elastically coupled chains oriented along the flow direction and a moving transverse smectic with decoupled flow chains. Such structures and the dynamic transitions between them have been extensively studied in a number of numerical simulations. ${ }^{5-7}$

Experimentally, a diversity of flow states has been reported in direct imaging experiments on $\mathrm{NbSe}_{2}$ crystals. $^{8-10}$ However, quantitatively the effect of pinning strength and/or temperature on the ordering velocity has been studied only through dc transport experiments, ${ }^{11-13}$ based on the assumption that an inflection point in dc current-voltage (IV) curve (i.e., a peak in differential resistance) marks the dynamic transition. Recently, different explanations have been given as the reason for such inflection point, like macroscopic coexistence of two phases ${ }^{14}$ and a change in the self-organized, large scale morphology of vortex rivers. ${ }^{15}$ Thus, a more direct, microscopic probe is required to study systematically the velocity, magnetic field, and temperature dependence of dynamic ordering.

Recently, we reported on the use of mode-locking (ML) experiments as a direct probe of ordering. ${ }^{16}$ The ML phenomenon occurs due to coupling between, on the one hand, collective lattice modes of frequency $f_{\text {int }}=q v_{\mathrm{dc}} / a$, with $q$ an integer and $a$ the lattice periodicity, which occur when a VA moves coherently with velocity $v_{\mathrm{dc}}$ through a pinning potential, ${ }^{10,17}$ and, on the other hand, a superimposed rf-drive of frequency $f$ at an integer fraction $1 / p$ of $f_{\text {int }}$. This coupling produces steps in the dc-transport $(I V)$ curves when $v_{\mathrm{dc}}$ $=(p / q) f a,{ }^{7,18-21}$ similar to ML steps in sliding charge density waves (CDW's) (Refs. 22,23) and giant Shapiro steps in Josephson junction arrays. ${ }^{24}$ However, on decreasing the velocity $v_{\mathrm{dc}}$ or increasing the temperature, incoherent fluctuations and plastic "events" due to quenched and thermal disorder reduce the width of the ML steps compared to that of an elastically moving system. Approaching the regime of fully plastic or liquid flow, the ML amplitude eventually vanishes $^{7,16}$ and the ML frequency $f_{c}$ at which this occurs provides a direct measure of the ordering velocity $v_{c}=f_{c} a$.

The particular system of our studies consists of mesoscopic flow channels in a disordered, strong pinning environment. ${ }^{21,25}$ The geometry of the samples is sketched in Figs. 1(a), 1(b). Vortices inside the channels are confined by strongly pinned vortices in the channel edges (CE's). When a force is applied along the channel, the shear interaction with these $\mathrm{CE}$ vortices provides the dominant pinning mechanism impeding the channel flow. Further, since the natural lattice (row) spacing is $a_{0} \simeq 1.075 \sqrt{\Phi_{0} / B}\left(b_{0}=\sqrt{3} a_{0} / 2\right)$, on varying the magnetic field $B$ one can go through a series of structural transitions from $n$ to $n \pm 1$ vortex rows in the channel (typically $n \lesssim 10$ ). In addition to its relevance for the study of (dynamic) structural transitions of vortex matter in quenched disorder, the physics of this system is also closely related to layering transitions in confined fluids, flow of colloids in mesopores and mesoscopic friction.

In Ref. 21 we have given a short account of the interesting phenomena which this system displays. First, due to the structural transitions, the dc-critical current for channel flow (yield strength) oscillates with field as shown in Fig. 1(e). At a given field, the dc $I V$ (force-velocity) curve with superimposed rf current exhibits the ML effect, as shown in Fig. 1(c). The ML condition in this case attains a form which is particularly useful to study the structural transitions. The voltage $V_{1,1}$ at which the fundamental $(p=q=1)$ ML step occurs is given by ${ }^{21}$

$$
V_{1,1}=f \Phi_{0} n N_{\mathrm{ch}}
$$



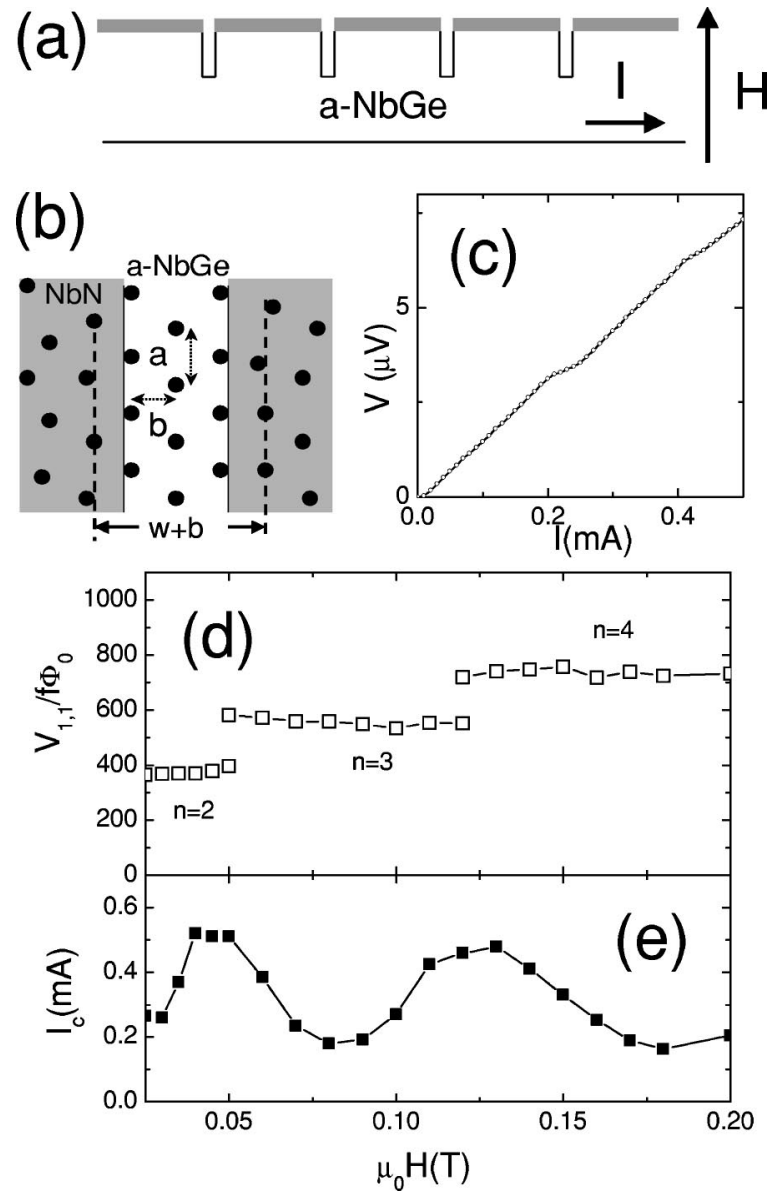

FIG. 1. (a) The channel device (side view) consisting of strong pinning $\mathrm{NbN}$ (dark) and weak pinning amorphous NbGe. The current and field directions are indicated. (b) Sketch of the vortex structure around a channel (top view). The effective channel width $w$ is also indicated. (c) Typical ML step in a dc- $I V$ curve at $70 \mathrm{mT}$ and a superimposed rf current of $3 \mathrm{MHz}$. (d) Normalized ML voltage $V_{1,1} /\left(f \Phi_{0}\right)$ versus magnetic field. (e) Critical current $I_{c}$, determined from a $1 \mu \mathrm{V}$ criterion, versus field.

with $\Phi_{0}$ the flux quantum, $N_{\text {ch }}$ the number of channels measured simultaneously, and $n$ the number of coherently moving chains in each channel.

As observed in Fig. 1(d), on changing field, $V_{1,1}$ increases as a staircase, directly reflecting the evolution of $n$ with field. A comparison with Fig. 1(e) shows that at mismatch fields, i.e., where a transition $n \rightarrow n \pm 1$ occurs and $n$ and $n \pm 1$ steps may coexist, the yield strength $\propto I_{c}$ is maximum, whereas around the center of the $V_{1,1}$ plateau, where an $n$ row configuration matches the channel width, it is minimum. As shown in Ref. 21, this phenomenon is caused by positional disorder (roughness) of the vortex configuration in the CE's: first of all, on moving away from the matching field this disorder enhances transverse fluctuations of vortex chains in the array, impeding the flow. Secondly, close to mismatch, part of the (moving) $n$-chain regions within each channel may switch to $n \pm 1$. In between the $n$ and $n \pm 1$ regions quasistatic fault zones with misaligned dislocations develop where the vortex trajectories are jammed. We note that the presence of degrees of freedom transverse to the average flow velocity, both in our system and vortex lattices in general, forms an important difference with CDW's. Particularly, for CDW's the "displacement" (phase) field is a scalar $\phi(x)$ and the velocity $\propto \partial_{t} \phi(x)$ represents longitudinal motion only. Our system, when compared to regular vortex lattices, has the unique property that the transverse response can be tuned.

In this paper we study in detail the ML step width as a function of the rf amplitude and frequency in detail. The experiments provide important information on the dynamic coherence of the arrays and how this coherency varies with mismatch and flow velocity. We focus on ML in the regime where $n=2 \rightarrow 3 \rightarrow 4$, but our findings are representative for other transitions with limited $n \lesssim 10$. The paper is organized as follows. In Sec. II we first review previous theoretical results on ML at high frequency and then present simulation results of a one-dimensional (1D) vortex chain to show the full frequency dependence of ML in a system with only elastic deformations. In Sec. III we describe the details of our sample and the experimental procedure. The experimental results are presented in Sec. IV. We find clear evidence for an ordering frequency $f_{c}$ below which no ML occurs. Furthermore, from the ML data we extract the pinning frequency $f_{p}$ and the total length $L_{\mathrm{ML}}$ of coherently moving three row regions. These quantities systematically change with magnetic field: at matching, where $L_{\mathrm{ML}}$ is large, $f_{p}$ and $f_{c}$ are both small, while at mismatch where $L_{\mathrm{ML}}$ is small, both $f_{p}$ and $f_{c}$ are enhanced. We find that $f_{c} \propto f_{p}^{2}$, independent of magnetic field. In Sec. V we compare these results with the ordering theory of Koshelev and Vinokur, and discuss the implications for the microscopic nature of the flow and the ordering phenomena.

\section{THEORETICAL CONSIDERATIONS}

The velocity (or frequency) dependence of the ML step width is particularly useful as a direct probe of dynamic ordering. At present, the ML step width has been studied theoretically only in the high frequency limit, where perturbation theory allows us to obtain an analytical description. ${ }^{26-30}$

\section{A. Equation of motion}

The 2D displacement field $\vec{u}(\mathbf{r}, t)$ of an elastic vortex lattice driven through a pinning environment by combined $\mathrm{rf}$ and dc external forces is (at $T=0$ )

$$
\gamma \frac{\partial \vec{u}}{\partial t}=\mathbf{F}_{D}+\mathbf{F}_{R}+\mathbf{F}_{P}
$$

with $\gamma=\Phi_{0} B / \rho_{f}$ the friction coefficient with $\rho_{f}$ the flux flow resistivity. $\mathbf{F}_{D}$ is a driving force per unit length consisting of dc and rf terms; $\left|\mathbf{F}_{D}\right|=j_{\mathrm{dc}} \Phi_{0}+j_{\mathrm{rf}} \Phi_{0} \cos (2 \pi f t)$ with $j_{\mathrm{dc}}$ and $j_{\text {rf }}$ the dc and rf current densities, respectively. $\mathbf{F}_{R}$ is the elastic restoring force given by $\left(\Phi_{0} / B\right)\left[\left(c_{11}-c_{66}\right) \nabla(\nabla \cdot \vec{u})\right.$ $\left.+c_{66} \nabla^{2} \vec{u}\right]$ with $c_{11}$ and $c_{66}$ the compression and shear moduli, respectively. ${ }^{1,5}$ In absence of the pinning force $\mathbf{F}_{P}$, the lattice is undistorted and flows uniformly: $d u / d t$ 
$=\mathbf{F}_{D} / \gamma=v_{\mathrm{dc}}+v_{\mathrm{ac}} \cos (2 \pi f t)$ with an ac velocity $v_{\mathrm{ac}}$ $=j_{\mathrm{rf}} \Phi_{0} / \gamma$, i.e., proportional to the rf drive.

\section{B. Amplitude of the ML-interference step}

At high velocities where the friction term dominates the pinning term in Eq. (2.1), one can treat the pinning as a perturbation with respect to the undisturbed $\mathrm{rf}$-dc velocity $\left|\mathbf{F}_{D}\right| / \gamma$. We distinguish two cases, namely, a periodic pinning potential and a random pinning potential. In case of a periodic potential with periodicities equal to that of the lattice, elastic deformations are absent $\left(\mathbf{F}_{R}=0\right)$ and the whole lattice behaves as a single particle with overdamped dynamics in a sinusoidal potential. At large drive this case is described analogous to a voltage biased, resistively shunted Josephson junction: ${ }^{31}$ substituting $u=v t$ in Eq. (2.1) and assuming $F_{P}$ $=\mu \sin (k u)$ with $\mu$ the maximum slope of the potential and $k=2 \pi / a$, one can show that as first order correction a step anomaly appears in the dc velocity-force characteristics at the ML condition $v_{\mathrm{dc}}=p a f$. The current density width of the $p$ th step oscillates with the rf drive according to

$$
\Delta j_{p, 1}=2 j_{c}\left|J_{p}\left(v_{\mathrm{ac}} / f a\right)\right|
$$

with $j_{c}=\mu / \Phi_{0}$ the critical current density and $J_{p}$ the Bessel function of the first kind of order $p$. Note that no subharmonic ML $(q \geqslant 2)$ occurs in this model.

Turning to a VL in a purely random potential, the first order perturbation correction has zero mean. The second order correction is the lowest order of perturbation that provides the ML step. Taking into account the lattice distortions due to the random pinning within the elastic limit, Schmidt and Hauger ${ }^{26}$ showed that the ML step can appear at both harmonic and subharmonic ML conditions $v_{\mathrm{dc}}=(p / q)$ af and that the associated width of the current density step is

$$
\begin{gathered}
\Delta j_{p, q}=2 \tilde{j}_{c}(q k) J_{p}^{2}\left(q v_{\mathrm{ac}} / f a\right), \\
j_{c}=\sum_{q} \tilde{j}_{c}(q k),
\end{gathered}
$$

where $\tilde{j}_{c}(q k)$ is the component of the critical current density related to the Fourier transform of the random potential correlator at wave vector $q k$. Thus, for random pinning the ML step width exhibits a squared Bessel-function oscillation with the rf drive. The same conclusion was obtained in a perturbation theory of CDW's in presence of $\mathrm{rf}$ and dc drive. ${ }^{29}$ In the following we omit the subscripts $p$ and $q$ in the ML step width since we will discuss only the fundamental ML phenomenon for $p=q=1$ (where $q=1$ will be justified in Sec. IV). We note here that in our case $a$ can be a frustrated lattice spacing different from the natural one in Ref. 26.

\section{Frequency dependence}

It is clear in Eqs. (2.2) and (2.3) that the dependence on frequency and $\mathrm{rf}$ drive only appear in the argument $z$ $=v_{\mathrm{ac}} / f a$ of the Bessel functions, irrespective of the type of pinning. Choosing $z$ by varying the rf amplitude such that
$J_{1}(z)$ is maximum, the characteristic maximum value of the fundamental ML width at high frequency is

$$
\begin{gathered}
\Delta j_{\max , P}=1.16 j_{c}, \\
\Delta j_{\max , R}=0.67 \tilde{j}_{c}(k)
\end{gathered}
$$

for periodic and random pinning, respectively.

These perturbational results are only applicable for frequencies (much) above the so-called pinning frequency $f_{p}$. For a sinusoidal pinning potential, $f_{p}$ is analogous to the characteristic frequency of an overdamped Josephson junction and it is given by

$$
f_{p} \equiv j_{c} \Phi_{0} / \gamma a \text {. }
$$

For random pinning, the fundamental ML step involves only the $k=2 \pi / a$ Fourier component of the pinning force $\widetilde{F}_{p}(k)=\tilde{j}_{c}(k) \Phi_{0}$ since it is responsible for the dynamic lattice mode excited at the washboard frequency $\left(f_{\text {int }}\right.$ $\left.=v_{\mathrm{dc}} / a\right)$. In this case one can define the pinning frequency $f_{p}$ (Ref. 32) such that the strength of the friction term $\gamma a f$ in Eq. (2.1) equals $\widetilde{F}_{p}(k)$ :

$$
f_{p} \equiv \widetilde{j}_{c}(k) \Phi_{0} / \gamma a .
$$

Below $f_{p}$ no analytical result for $\Delta j_{\max }$ is available, not even for sinusoidal pinning. ${ }^{33}$ In this regime numerical simulations are a useful tool to obtain the theoretical value of the fundamental ML width. ${ }^{34,35}$ To obtain $\Delta j_{\max }$ vs $f$ for the case of completely elastic motion in our channel system, we have performed molecular dynamics simulations of an rf-dc driven 1D elastic vortex chain both in a channel with periodically configured static vortices in the CE's and in channels with strongly disordered CE vortex arrangements [see the inset of Fig. 2(c) and Refs. 36,37 for more details]. The channel width is $w=b_{0}$, i.e., the (average) spacing between the first pinned rows is $2 b_{0}$. Vortex interactions were modelled by the London potential with $\lambda / a_{0}=1$ ( $\lambda$ is the penetration depth) and the average vortex spacing $a$ in the channel was chosen equal to that in the CE's, $a=a_{0}$.

For the periodic case, the CE potential is sinusoidal and the critical current density $j_{c}$ is given by its maximum slope $j_{c}=\mu / \Phi_{0}$. Simulating a chain of limited length was sufficient since all vortices behave as a single particle. Figure 2 shows an example of the ML step for superimposed $\mathrm{rf}$ drive of amplitude $v_{\mathrm{ac}} /(f a)=2$ and frequency $f \simeq 3 f_{p}$. In Fig. 2(c), we summarize the numerical results of $\Delta j_{\max , P}$ versus frequency, represented by the solid squares. Here, $\Delta j_{\max , P}$ is normalized by $j_{c}$ and the frequency is normalized by $f_{p}$ defined by Eq. (2.7). At high frequency $f>f_{p}, \Delta j_{\max , P}$ saturates at a frequency independent value $\Delta j_{s, P} / j_{c} \simeq 1.13$ very close to the theoretical prediction Eq. (2.5) for periodic pinning. For smaller $f, \Delta j_{\max , P}$ starts to decrease around $f_{p}$ and then vanishes linearly with $f$. The whole frequency dependence of $\Delta j_{\max , P}$ is well approximated by an empirical function

$$
\Delta j_{\max }=\Delta j_{s} \tanh \left(f / 0.7 f_{p}\right)
$$



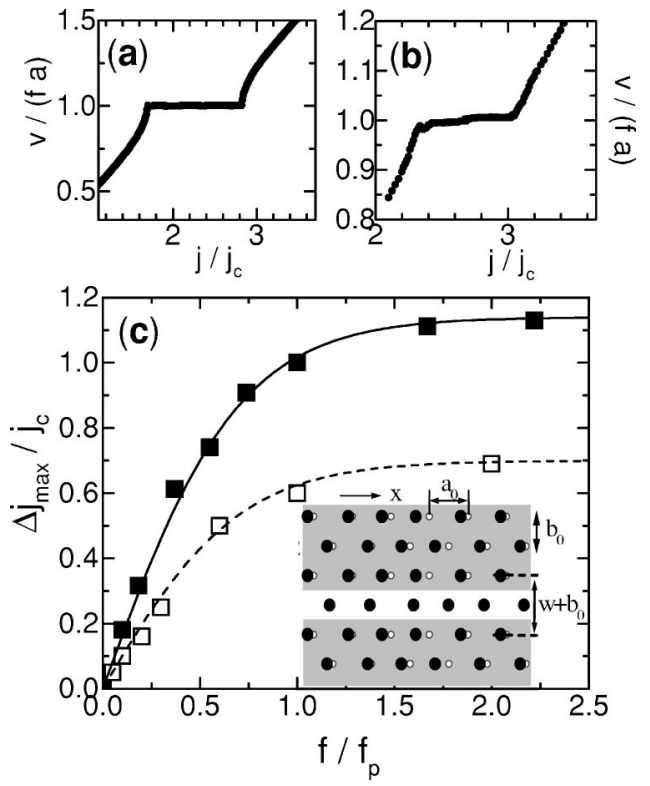

FIG. 2. (a) Normalized force-velocity curves for simulations of a vortex chain with an $\mathrm{rf}$ drive of frequency $f=3 f_{p}$ and amplitude $v_{\text {ac }} /(f a)=2$ in a periodic 1D channel; (b) same as (a) in a disordered 1D channel. (c) Maximum ML current density step width from simulations with $\mathrm{rf}$ drive of various frequencies and amplitudes: ( $\square$ ) results for a channel with periodic CE vortices; ( $\square$ ) results for disordered $1 \mathrm{D}$ channels. The curves show the empirical relation Eq. (2.9) with $C=1.16$ (drawn line) and $C=0.69$ (dashed line). The inset shows the simulation geometry for a periodic $\mathrm{CE}$ configuration $(\bigcirc)$ and for a disordered CE configuration $(\bigcirc)$.

in which we have omitted the subscript referring to the periodic pinning potential. Equation (2.9) is represented by the solid line in Fig. 2(c).

For the disordered 1D channel, the CE vortices are assigned relatively large random shifts over distances $|\mathbf{d}|$ with $\sqrt{\left\langle(\nabla \cdot \mathbf{d})^{2}\right\rangle}=0.12$ with respect to the regular lattice configuration. ${ }^{37,38}$ We used a chain of 2000 vortices for which the results have become length independent. Due to the disorder, both the numerically obtained threshold force $\propto j_{c}^{R}$ and the step width $\Delta j_{\max , R}$ are strongly reduced [by a factor $\sim 5$ (Ref. 37)] with respect to the ordered case. An example of the simulated ML step is shown in Fig. 2(b). There are two distinct differences with the periodic case, displayed in Fig. 2(a): (i) the sharp corners disappear and (ii) both below and above the ML condition the curve in (b) is essentially linear with the same slope, with a shift at the ML condition, very similar to the experimental result in Fig. 1(c). After normalization $\Delta j_{\max , R} / j_{c}^{R}$ and $f / f_{p}=\gamma a f /\left(j_{c}^{R} \Phi_{0}\right)$, we plot the simulation results collected for various frequencies in Fig. 2(c) as the open squares. The saturation value $\Delta j_{\max , R}\left(f \gg f_{p}\right) \equiv \Delta j_{s, R} \simeq 0.7 j_{c}^{R}$, very close to the result in the random pinning limit in Eq. (2.6). The whole frequency dependence is then again well approximated by Eq. (2.9) [dashed line in Fig. 2(c)] in which we have now implicitly assumed the subscript $R$ referring to the quantities in the random case.

It is worth mentioning that the results for the disordered channel were insensitive to small changes in the ratio $a / a_{0}$.
Further, we note that both in the periodic and the disordered channel simulations ML can be observed down to $f=0$. We believe this is a direct consequence of the fact that vortices in the chain remain elastically connected, because other simulations in which also transverse degrees of freedom and plasticity are allowed, do not show this feature. ${ }^{39}$

\section{EXPERIMENT}

The device consists of a strong pinning layer of polycrystalline $\mathrm{NbN}$ film on top of a weak pinning amorphous $(a-)$ $\mathrm{Nb}_{1-x} \mathrm{Ge}_{x}$ film $(x \approx 0.3)$. The thickness of the $\mathrm{NbN}$ and $a$-NbGe films are 50 and $550 \mathrm{~nm}$, respectively. Using reactive ion etching with proper masking ${ }^{40}$ narrow straight channels were etched from the top $(\mathrm{NbN})$ layer leaving a $300 \mathrm{~nm}$ $\left(=d_{\mathrm{ch}}\right)$ thick $(a-\mathrm{NbGe})$ bottom layer, see Fig. 1(a). The width and length of each channel are $230 \mathrm{~nm}$ and $300 \mu \mathrm{m}$ $(=L)$, respectively. The spacing between adjacent channels is $10 \mu \mathrm{m}$. Magnetic field was applied perpendicular to the films, inducing a vortex array as schematically shown in Fig. 1(b). The transport current was applied perpendicularly to the channel, providing a driving force parallel to the channel.

For the ML measurement, we recorded dc voltage by sweeping the dc current with superimposed rf current. The transmission lines for the rf current were terminated by matching circuits very close to the sample. To avoid heating, both the sample and the circuits were immersed in superfluid ${ }^{4} \mathrm{He}$. For consistency, all the data presented in this paper were taken after field cooling in which the magnetic field was applied above $T_{c}$ 's of $a$-NbGe $(2.68 \mathrm{~K})$ and $\mathrm{NbN}(11 \mathrm{~K})$ and the sample subsequently cooled to $T=1.9 \mathrm{~K}$, which is much lower than the vortex lattice melting temperature $\approx 2.5 \mathrm{~K}$ for the fields we studied. The ML steps in $I V$ curves are always rounded as in Fig. 1(c). For definition of the current step width $\Delta I$, we took the derivative of the $I V$ curve and integrated over the ML peak in the differential conductance-voltage curve with respect to the flux-flow base line. $^{21}$

\section{RESULTS}

Our measurements were carried out in the magnetic field regime where three-chain structures exist in the channels, ranging from 45 to $110 \mathrm{mT}$, see Fig. 1(d). We thus focus on the fundamental ML step characterized by $V_{1,1} /\left(\Phi_{0} f N_{\mathrm{ch}}\right)$ $=3$ originating from coherently moving $n=3$ regions. At the borders of our field range coexistence with $n=2$ or $n=4 \mathrm{ML}$ steps may occur.

In Fig. 3 we show an example of how the fundamental ML width $\Delta I$ depends on the rf drive $I_{\mathrm{rf}}$ for $\mu_{0} H=80 \mathrm{mT}$ and $f=12 \mathrm{MHz}$. $\Delta I\left(I_{\mathrm{rf}}\right)$ shows oscillatory behavior with a maximum value $\Delta I_{\max }$ in the first lobe, as indicated in the figure. A qualitative comparison of the data with the theoretical predictions Eq. (2.3) (solid line) and Eq. (2.2) (dotted line) shows that the data follows more closely a $J_{1}^{2}$ than a $\left|J_{1}\right|$ dependence. At $\Delta I_{\max }$ it is found that $I_{\mathrm{rf}}=(1.9$ $\pm 0.3) I_{\mathrm{dc}}$ over a broad frequency range between $f_{p}$ and 40 $\mathrm{MHz}$ (above this frequency the experimental error in $I_{\mathrm{rf}}$ becomes larger). This value is in good agreement with Eq. (2.3) 


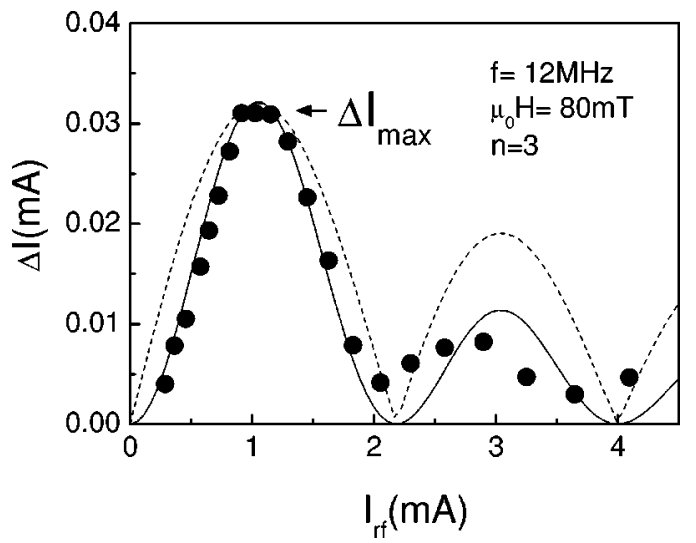

FIG. 3. Current width $\Delta I$ of the fundamental ML step vs $\mathrm{rf}$ current $I_{\mathrm{rf}}$ taken at $12 \mathrm{MHz}$ and $80 \mathrm{mT}$. The maximum value $\Delta I_{\max }$ is indicated. Solid and dotted curves display $\Delta I \propto J_{1}^{2}(z)$ and $\Delta I$ $\propto\left|J_{1}(z)\right|$, with $z \propto I_{\mathrm{rf}}$, respectively.

which has a maximum at $z=v_{\mathrm{ac}} / f a=v_{\mathrm{ac}} / v_{\mathrm{dc}} \approx I_{\mathrm{rf}} / I_{\mathrm{dc}}=1.8$. It is important to note that while the values of the rf current might appear rather large, the actual vortex displacements due to the $\mathrm{rf}$ drive at or below the first maximum in $J_{1}^{2}(z)$, are less than $1.8 /(2 \pi) \simeq 0.3$ of the lattice spacing.

The $J_{1}^{2}(z)$ behavior shows that the pinning potential due to the vortices in the $\mathrm{CE}$ acts as a random potential (RP). ${ }^{41}$ The origin of the RP is the strong positional disorder of the vortex lattice in the $\mathrm{NbN}$ edge material. This disorder has recently been observed in scanning tunnelling microscopy experiments on $\mathrm{NbN}$ films. ${ }^{42}$ We further note that we did not observe subharmonic ML steps, i.e., there were no ML steps at $V_{\mathrm{ML}}=3 f \Phi_{0} N_{\mathrm{ch}} / q$ with $q \geqslant 2$. In the context of our RP this seems in contradiction to the results of others. ${ }^{18}$ But those experiments have been carried out at relatively low fields where the RP has short range correlations on a scale $\xi \ll a$. Fourier components $q k$ with $q \geqslant 2$ are needed to describe such short range fluctuations and therefore subharmonic ML steps are seen. In our case, the RP is due to the $\mathrm{CE}$ vortices which have average spacing $a_{0} \simeq a$. Consequently the most important Fourier component describing this RP is the $q=1$ mode, which explains why we do not see subharmonic ML steps.

Next we turn to the frequency dependence of $\Delta I_{\max }$. Figure 4 shows $\Delta I_{\max }(f)$ at $50 \mathrm{mT}$ obtained from measurements similar to those in Fig. 3 at various frequencies. As in the numerical results in Fig. 2(c), $\Delta I_{\max }$ saturates at a value $\Delta I_{s} \simeq 78 \mu \mathrm{A}$ at high frequencies, while at low frequencies it decreases monotonically with $f$. A large part of the data is well approximated by the empirical function discussed in the previous section, $\Delta I_{\max }=\Delta I_{s} \tanh \left(f / 0.7 f_{p}\right)$, and we can extract the pinning frequency $f_{p}=7.8 \mathrm{MHz}$ as the remaining fit parameter. However, at low frequency (i.e., small dc velocity) the data lie significantly below the empirical curve. This implies that the vortex motion becomes less coherent due to the disordered CE's. On reducing $f, \Delta I_{\max }$ vanishes almost linearly at a finite frequency $f_{c}$ determined by the intersection between the dotted line and the $\Delta I_{\max }=0$ axis. In this regime, the rf current for which $\Delta I$ exhibits its maximum value, starts to saturate at a value $\sim I_{c} .{ }^{43}$ The collapse of

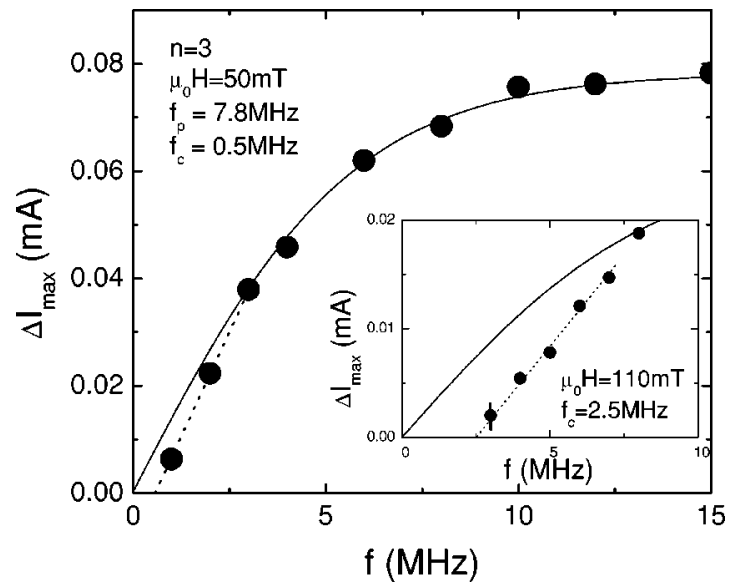

FIG. 4. The maximum current width $\Delta I_{\max }$ as a function of frequency $f$ at $50 \mathrm{mT}$. The solid curve shows the fit according to the empirical function Eq. (2.9). The dotted line shows the linear extrapolation to $\Delta I_{\max }=0$ for definition of the dynamic ordering frequency $f_{c}$. The inset shows the onset behavior of $\Delta I_{\max }$ and ordering frequency $f_{c}$ for a field of $110 \mathrm{mT}$. The solid curve shows a fit of the high frequency data to Eq. (2.9).

$\Delta I_{\max }$ at finite frequency $f_{c}$ is even more clearly visible in the data taken at $110 \mathrm{mT}$ shown in the inset to Fig. 4. Below $f_{c}$ no ML step appears at any rf drive, indicating the complete absence of coherent three-row motion.

The above fitting analysis was performed on $\Delta I_{\max }(f)$ data taken at various fields in the $n=3$ field regime and we extracted $f_{p}, \Delta I_{s}$ and the dynamic ordering frequency $f_{c}$. We first discuss the results of $f_{p}$ and $\Delta I_{s}$ as a function of magnetic field, shown in Fig. 5(b). As observed, $f_{p}$ has a minimum at $70 \mathrm{mT}$, somewhat below the middle of the plateau in $V_{1,1}$ in Fig. 5(a), and it increases on approaching either end of the plateau. Hence, the associated pinning current density in the coherent three-chain regions $\tilde{j}_{c}(k)$ is small at $70 \mathrm{mT}$ and increases away from $70 \mathrm{mT}$. In fact, the value of $\tilde{j}_{c}(k)$ as determined from $f_{p}$ using Eq. (2.8) agrees within $30 \%$ with $j_{c}$ as determined directly from dc- $I V$ curves, Fig. 1(e). Meanwhile, $\Delta I_{s}$ exhibits a field dependence which differs considerably from that of $f_{p}$ : it has a broad peak around $B$ $=50 \mathrm{mT}$ and then decreases with increasing field. Clearly, this behavior cannot be explained by simply assuming $\Delta I_{s}(B) \propto j_{c}(B) \propto f_{p}(B)$.

At this point we note that theory assumes all vortices in the channel are moving coherently. However, in our experiment only a fraction of the vortices move coherently. Specifically, an $n$-row region may locally break up due to the strong edge disorder or it may coexist with $n \pm 1$-row regions (with different ML voltages) due to mismatch. ${ }^{21}$ We define the total length of mode-locked regions with three coherently moving rows as $L_{\mathrm{ML}}$ and the mode-locked fraction as $L_{\mathrm{ML}} / L$. Since only the coherent $n$-row regions contribute to $\Delta I_{s}$, we can link the value of $\Delta I_{s}$ to that of $\Delta j_{s}$ by using $\Delta I_{s}=L_{\mathrm{ML}} d_{\mathrm{ch}} \Delta j_{s}$. In this expression $\Delta j_{s}$ can be obtained from the measured pinning frequency via Eqs. (2.8) and (2.6). Using $\gamma=\Phi_{0} B / \rho_{f}$ the result for $L_{\mathrm{ML}}$ is given by 


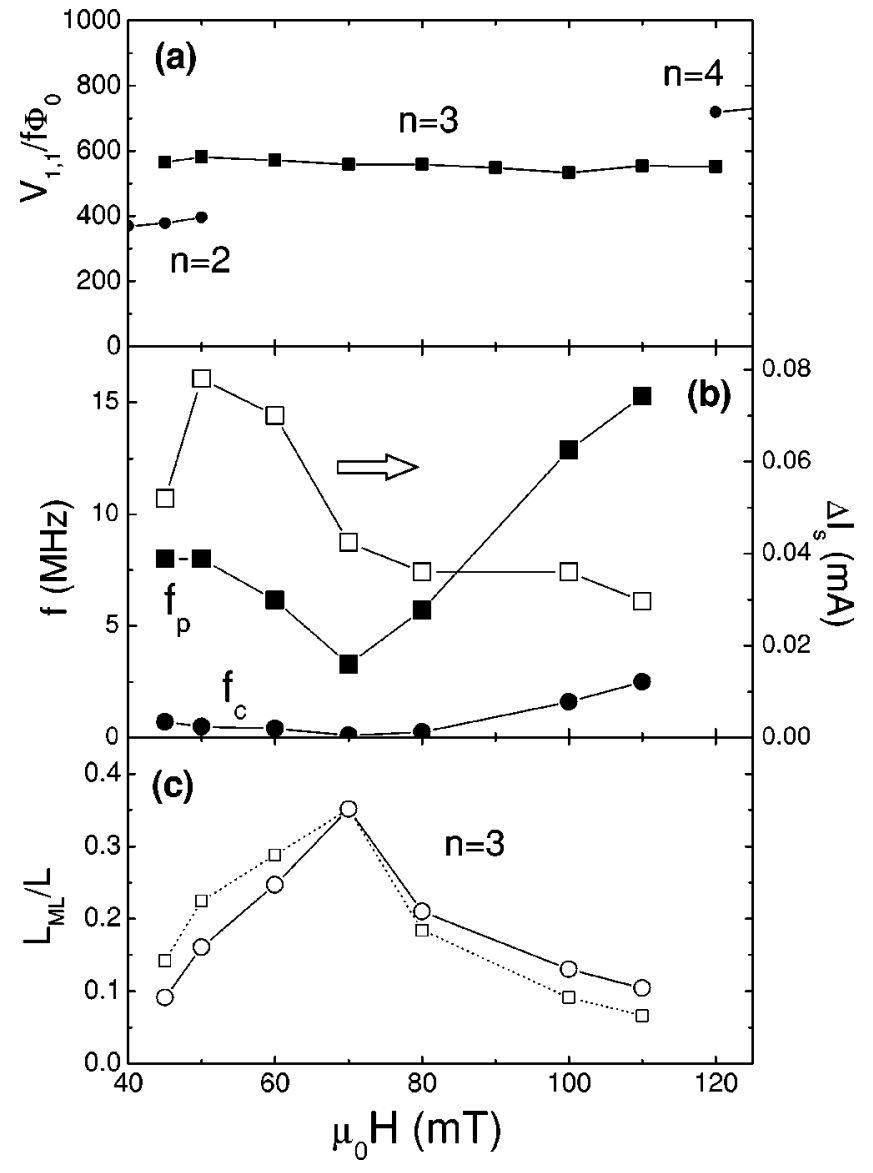

FIG. 5. (a) $V_{1,1} / f \Phi_{0}$ versus field. (b) The pinning frequency $f_{p}$ $(\boldsymbol{\square})$, the dynamic ordering frequency $f_{c}(\boldsymbol{O})$, and the saturation value of the maximum current width $\Delta I_{s}(\square)$ for $n=3$ as a function of field. The data are obtained from fits to the measured $\Delta I_{\max }(f)$. (c) The coherently moving fraction $L_{\mathrm{ML}} / L$ of $n=3$ regions vs field, where $L_{\mathrm{ML}}$ is determined from Eq. (4.1), with $a$ $=a_{0}(B)(\square)$ and $a=a_{M} B_{M} / B(\bigcirc)$, where the subscript $M$ refers to the matching field, see text. All lines are guides to the eye.

$$
L_{\mathrm{ML}}=\frac{\Delta I_{s}}{f_{p} a} \frac{\rho_{f}}{0.67 B d_{\mathrm{ch}}} .
$$

The different field dependencies of $\Delta I_{s}$ and $f_{p}$ mentioned above should thus be attributed to an additional field dependence of $L_{\mathrm{ML}}$.

We now evaluate $L_{\mathrm{ML}}$ using $\rho_{f}$ for amorphous $\mathrm{NbGe}$ films ${ }^{44-46}$ and first assume an equilibrium lattice spacing $a$ $=a_{0}\left(\simeq 1.075 \sqrt{\Phi_{0} / B}\right)$ with $B=\mu_{0} H$. Figure 5(c) shows $L_{\mathrm{ML}}$ normalized by the channel length $L$ vs field (square symbols). As observed, the coherently moving fraction is maximum at $B \simeq 70 \mathrm{mT}$. This provides a clear definition of the matching field $B_{M}$ for $n=3$. At $B_{M}$, the longitudinal spacing $a$ in the channel should obey $a \equiv a_{M}=a_{0}$ and the row spacing $b_{M}=\Phi_{0} / B_{M} a_{M}$ is commensurate with the effective channel width, i.e., $3 b_{M}=w$. However, away from the matching field the array will be frustrated (stretched or compressed) due to the confinement. In particular, for $B<B_{M}$ the lattice spacing $a>a_{0}$, while for $B>B_{M}, a<a_{0}$. The maximum possible difference between $a$ and $a_{0}$ would be

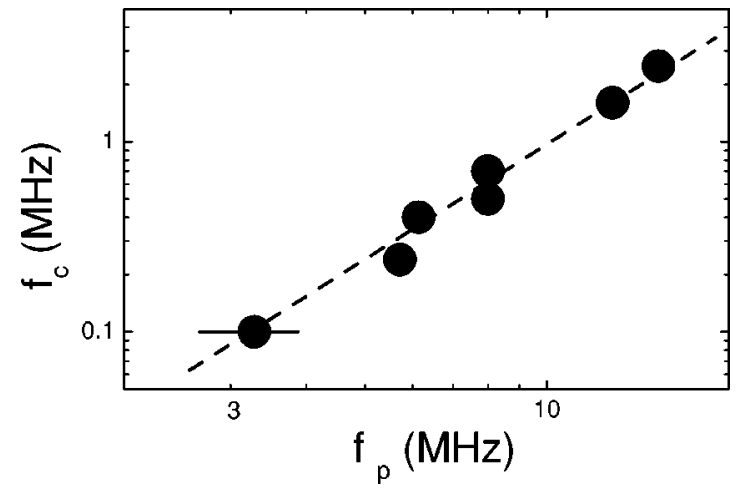

FIG. 6. Dynamic ordering frequency $f_{c}$ versus pinning frequency $f_{p}$. Dotted line: $f_{c}=\tau f_{p}^{2}$ with $\tau \simeq 1 \times 10^{-8} \mathrm{~s}$.

achieved when the row spacing $b$ would not change with mismatch, i.e., $b(B)=b_{M} \cdot{ }^{47}$ This would imply $a$ $=\left(B_{M} / B\right) a_{M}$. Inserting this relation for $a$ in Eq. (4.1), the result for $L_{\mathrm{ML}}$, shown by the open circles, is slightly modified but shows essentially the same behavior as our first analysis: upon increasing the frustration, which we define as $\left|1-\left(B / B_{M}\right)\right|$, the spatial extent of regions with three coherently moving rows shrinks progressively. An additional analysis of the $n=2 \mathrm{ML}$ steps which occur at lower fields ( $B \simeq 50 \mathrm{mT}$, where the transition $n=3 \rightarrow 2$ takes place), shows consistently that the spatial extent of the 2 row ML regions increases upon further decreasing field.

Finally we discuss the behavior of the ordering frequency $f_{c}$. As shown in Fig. 5(b), $f_{c}(B)$, denoted by $(\bigcirc)$, exhibits a minimum at the matching field and systematically increases with mismatch. Similarly to the decay of $L_{\mathrm{ML}}$ with increasing frustration, this shows that a larger mismatch progressively induces more disorder. We also find that, when the field is reduced below $B \simeq 50 \mathrm{mT}$, where a two-row configuration first appears, the ordering frequency $f_{c}^{n=2}$ for the emergence of the $n=2$ ML effect decreases.

Qualitatively, the behavior of $f_{c}$ is similar to that of the pinning frequency $f_{p}$ (or $I_{c}$ ). Both are important quantities characterizing the random pinning of a system and are not independent, as follows from a double logarithmic plot of $f_{c}$ as a function of $f_{p}$, shown in Fig. 6. The data are well described by the relation $f_{c}=\tau f_{p}^{2}$ with $\tau \simeq 1 \times 10^{-8} \mathrm{~s}$, represented by the dashed line. A more detailed fit of the data using a power law relation $f_{c} \propto f_{p}^{\alpha}$ yields an exponent $\alpha$ $=2.1 \pm 0.1$.

\section{ANALYSIS AND DISCUSSION OF DYNAMIC ORDERING AND DEPINNING THRESHOLD BEHAVIOR}

\section{A. Comparison with the KV theory}

For a proper discussion of the above results, we first shortly describe the phenomenological ordering theory of Koshelev and Vinokur (KV). ${ }^{1}$ In their study of a $2 \mathrm{D}$ vortex system with strong random bulk pinning, they found that the shaking action due to motion through the pinning potential can be represented by a "shaking temperature" $T_{\text {sh }}$ which 
decreases with velocity as $T_{\mathrm{sh}} \propto 1 / v$. The dynamic ordering transition occurs when the effective temperature $T+T_{\mathrm{sh}}$ is reduced below the equilibrium melting temperature $T_{m}$, i.e., when the velocity exceeds $v_{c} \propto 1 /\left(T_{m}-T\right)$. In later work ${ }^{4,48}$ it was shown that the shaking temperature refers to (bond) fluctuations transverse to the velocity and that the associated ordering at $v_{c}$ corresponds to so called transverse freezing, where interchain excursions (permeation modes) are strongly suppressed. Within the KV theory we can express the ordering frequency $f_{c}=v_{c} / a$ as

$$
f_{c}=\sqrt{3 / 2 \pi} \frac{\gamma_{u} \rho_{f}}{\Phi_{0}^{2} a^{2} d_{\mathrm{ch}} k_{B}\left(T_{m}-T\right)},
$$

with $\gamma_{u}$ the mean squared 2D pinning energy multiplied by the area of a pin, $\rho_{f}$ the flux flow resistivity, $a$ the lattice spacing and $d_{\mathrm{ch}}$ the film thickness.

In our channel system the ordering can also be described as transverse freezing. We observed this in simulations (e.g. for $w / b_{0} \simeq 3$ ) as a suppression of the interchain excursions in parts of the channel at sufficient velocity. ${ }^{39}$ However, different from the 2D system considered by $\mathrm{KV}$, these interchain excursions and the associated shaking temperature now arise from the random interaction with the disordered vortices in the CE's and a modification of Eq. (5.1) is required. Thinking in terms of bond fluctuations or a Lindemann criterion, as in Ref. 4, it is clear that it is the short wavelength $\sim a_{0}$ disorder component in the potential due to quenched vortex displacements $\mathbf{d}$ in the $\mathrm{CE}$ which is relevant for the "shaking temperature." This component acts only in a range $\sim a_{0} / 2$ from the first pinned row, ${ }^{39}$ therefore shaking of the outer rows should govern the transverse freezing. A rough estimate within London theory yields an r.m.s. amplitude of the random stress near the edge $\sim \varepsilon_{c e} c_{66}$ with $c_{66}$ the shear modulus and $\varepsilon_{c e} \simeq\left(\sqrt{\left\langle|\mathbf{d}|^{2}\right\rangle} / a_{0}\right) /(\pi \sqrt{3})$ representing the random strain. ${ }^{16,39}$ Taking the longitudinal range of a pin also to be $a_{0} / 2$, we replace the parameter $\gamma_{u}$ in Eq. (5.1) by $\gamma_{c e}$, resulting in

$$
\gamma_{c e} \simeq\left(\varepsilon_{c e} c_{66} a_{0} b_{0} d_{\mathrm{ch}}\right)^{2}\left(\frac{a_{0}}{2}\right)^{2}
$$

Further it is important to realize the following: the energy scale $k_{B} T_{m}$ in Eq. (5.1) should be regarded as the energy for creation of the dislocation pairs that are required for plastic motion, i.e., $k_{B} T_{m} \rightarrow k_{B} T_{p} \simeq c_{66} a_{0}^{2} d_{\mathrm{ch}} /(2 \pi)$, with $c_{66}$ evaluated at the field and temperature of the measurement. ${ }^{49}$ For our temperature and fields, this energy $k_{B} T_{p}$ is two orders of magnitude larger than the thermal energy $k_{B} T$ which we can therefore neglect in Eq. (5.1). Hence, the random shaking $(\propto 1 / v)$ in our case essentially represents "cold working" of the moving structure. We also anticipate that the energy $k_{B} T_{p}$ should depend on the matching condition since a reduction of this energy eventually drives the transition to $n=2$ or $n$ $=4$ rows. Therefore we add a mismatch dependent factor

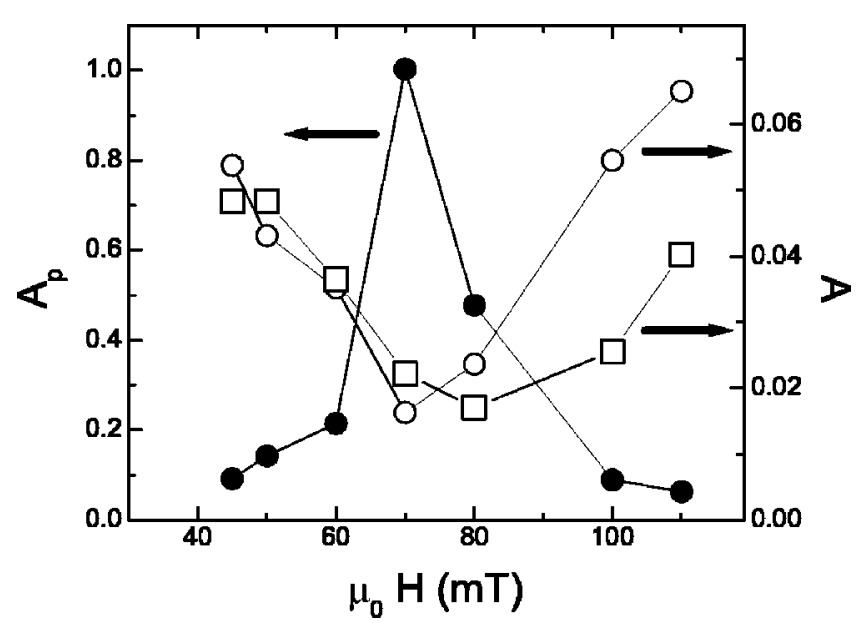

FIG. 7. (@) The parameter $A_{p}$ as determined from $f_{c}$ in Fig. 5(b) and Eq. (5.3) using $\varepsilon_{c e}=0.025$. ( $\square$ ) The parameter $A$ describing the pinning strength determined directly from Eq. (5.4) and the measured critical current density. $(\bigcirc) A$ determined from Eq. (5.5) using the field dependence of $A_{p}$ and $\tau=10^{-8} \mathrm{~s}$.

$A_{p}: k_{B} T_{p}=A_{p} c_{66} a_{0}^{2} d_{\mathrm{ch}} /(2 \pi)$, where $A_{p}$ is assumed to be 1 at matching. Taking into account these changes, Eq. (5.1) becomes

$$
f_{c} \simeq \frac{\sqrt{\pi} \varepsilon_{c e}^{2} c_{66} \rho_{f}}{2 A_{p} \Phi_{0} B} .
$$

Using the experimental parameters with $c_{66}$ $=\Phi_{0} B /\left(16 \pi \mu_{0} \lambda^{2}\right)$ and $\lambda(1.9 \mathrm{~K}) \simeq 1.1 \mu \mathrm{m}$, we obtain the value of $\varepsilon_{c e}$ from the value of $f_{c}$ at matching: $\varepsilon_{c e} \simeq 0.025$, i.e., $\sqrt{\left\langle|\mathbf{d}|^{2}\right\rangle} / a_{0} \simeq 0.13$. This is in very reasonable agreement with our estimate $\left(\sqrt{\left\langle|\mathbf{d}|^{2}\right\rangle} / a_{0} \simeq 0.10\right)$ near the melting temperature in Ref. 16. From Eq. (5.3) and the $f_{c}$ data in Fig. 5(b) we can also extract the field dependence of $A_{p}$ characterizing the reduction of the defect creation energy. The result is plotted in Fig. 7, showing that close to mismatch $A_{p}$ has decreased by an order of magnitude.

Next, we turn to the relation between $f_{c}$ and $f_{p}$. The dc-critical current density can be described phenomenologically by ${ }^{25}$

$$
j_{c}=2 A c_{66} /(B w),
$$

where in our case $A$ varies from $A \simeq 0.015$ at matching to $A \simeq 0.04-0.05$ at mismatch, see the open squares in Fig. 7 . Further, when we combine Eqs. (5.3), (5.4) with Eq. (2.8) and use $\gamma=\Phi_{0} B / \rho_{f}$, we obtain the quadratic relation $f_{c}$ $=v_{c} / a=\tau f_{p}^{2}$ observed experimentally in Fig. 6 with the time scale $\tau$ given by

$$
\tau \simeq 0.5\left(\varepsilon_{c e} / A\right)^{2} \frac{(w B)^{2}}{A_{p} c_{66} \rho_{f}} .
$$

We note that the relation $f_{c} \propto f_{p}^{2}$ is in fact a general result from the KV theory in the strong pinning limit. Considering Eq. (5.5), since $\rho_{f}, c_{66} \propto B$ and experimentally we found that $\tau \simeq 10^{-8} \mathrm{~s}$, independent of field, this implies that $A$ $\propto \sqrt{1 /\left(\tau A_{p}\right)}$. Using this relation with the field dependent 
value of $A_{p}$, we obtain $A$ as shown by the open circles in Fig. 7. The minima in both data are slightly shifted and deviations are seen for $B>B_{M}$, but given the approximations made, the overall agreement is still reasonable. An important physical implication of the relation $A \propto \sqrt{1 /\left(A_{p}\right)}$ is that the increase in pinning strength away from matching is directly related to the reduction of the defect creation energy. In other words, the rise in $A$ reflects an effective softening of the array in the channel upon increasing mismatch. Through this softening it is able to better adjust to the random CE pinning potential, very much analogous to the mechanism responsible for the peak effect in ordinary superconductors. ${ }^{50}$ In our case a more detailed picture of the softening mechanism is possible. We already mentioned that for $B>B_{M}$, the chains are longitudinally compressed $\left(a<a_{0}\right)$. This will facilitate deviations in the transverse direction and reduce the energy to (dynamically) create interstitials between rows. For $B$ $<B_{M}$, the chains are stretched, i.e., $a>a_{0}$. In this case the energy for a chain to accept vortices from a neighboring chain is lowered. This in turn facilitates a configurational change in which the array can better adopt to the CE potential. An additional mechanism for the rise in $A$ for $B<B_{m}$ is that, due to the mismatch, the outer chains will be pushed towards the $\mathrm{CE}$, leading to an enhanced influence of the $\mathrm{CE}$ potential.

To conclude this section, we shortly discuss the possible influence of "extrinsic" defects in our samples which may be a source of incoherency and limit $f_{c}$. Such "extrinsic" defects could consist of a $\mathrm{NbN}$ bridge over the channel or a physical edge roughness on a scale $\geqslant b_{0}$. First, when such defects are important to the behavior, this would rapidly destroy the critical current oscillations of the channels, the amplitude of which quantitatively agreed with that of samples with channels of different width. Secondly, extrinsic defects cannot explain the variation of $f_{c}$ with magnetic field nor its quadratic dependence on $f_{p}$ (or $j_{c}$ ). The quantitative agreement of our data with the modified KV theory decisively shows that the microscopic roughness of the pinned $\mathrm{CE}$ arrays forms the dominant (intrinsic) disorder in our system.

\section{B. dc versus dc+rf driven state}

So far we have tacitly assumed that the flow behavior obtained from our rf-dc measurements simply reflects that of the dc-driven structure. We now discuss to what extent the additional rf current itself influences the behavior. Recent measurements of the rf impedance, ${ }^{51}$ which is a sensitive probe of ML at small rf currents, have shown that on approaching the dc-driven state, i.e., when $I_{\mathrm{rf}} \rightarrow 0$, the voltage broadening $\delta V_{1,1}$ of the fundamental ML step diverges. Since $\delta V_{1,1} \propto \delta f_{\text {int }}$, this broadening reflects fluctuations in the washboard frequency and, via $f=v / a$, fluctuations in the velocity and in the longitudinal lattice spacing $a .{ }^{52}$ Correspondingly, the divergence of $\delta V_{1,1}$ implies that the dcdriven state lacks temporal coherence. We also did not observe any narrow band noise in the voltage spectrum of the dc-driven state (even for only 30 channels at large dc drive). At the same time, $V_{1,1} \propto n$ remains constant for $I_{\mathrm{rf}} \longrightarrow 0$, from which we conclude that the dc state still exhibits local regions organized in three moving chains. Thus, at sufficient velocity the dc state would correspond to temporally incoherent, confined smectic regions ${ }^{3}$ of finite (mismatch dependent) length, with liquidlike intrachain order and residual interchain excursions.

In presence of rf current the fluctuations are strongly reduced, as also observed in experiments on CDW's. ${ }^{3}$ In simulations we observed that the suppression of the interchain excursions plays an important role in this process, causing transversely frozen regions in the channel. However, the rf-dc $I V$ curves always show incomplete ML with the same broadening $\delta V_{1,1}$ as discussed above. This broadening is too large to be explained by the elastic theory in Ref. 26 . Therefore, it is either caused by residual slip in the $n$-row regions or by remaining plastic regions with interconnecting rows, but further experimental and numerical work is required to decide on this issue.

Finally, we shortly return to the frequency dependence of the ML current width $\Delta I_{\max }$ in Fig. 4. Extending the relation $\Delta I_{\max }(f) \propto \Delta j_{\max }(f) L_{\mathrm{ML}}(f)$ to frequencies below $f_{p}$ and taking the ideal $\tanh (f)$ behavior Eq. (2.9) for $\Delta j_{\max }$, we find that the ordering frequency $f_{c}$ would mark the velocity where $L_{\mathrm{ML}} \rightarrow 0$. Such interpretation implies that the dynamic ordering in our disordered system is a smooth, second order dynamic phase transition.

\section{SUMMARY}

Using mode-locking experiments, we have investigated the dynamics of vortex arrays confined in disordered mesoscopic channels. The ML effect allows us to trace in detail structural transitions from $n-1 \rightarrow n \rightarrow n+1$ confined moving vortex chains on changing field. A study of the amplitude and frequency dependence of the ML steps and comparison to simulations of an elastic chain provide a complete characterization of the pinning strength, dynamic ordering velocity and coherency of the arrays. We find that the spatial extent $L_{\mathrm{ML}}$ of coherently moving $n$ row regions is large at a matching field and shrinks with increasing mismatch. At the same time both the pinning frequency $f_{p} \propto j_{c}$ and the ordering frequency $f_{c}$ (proportional to the ordering velocity) increase with mismatch. We show that $f_{c} \propto f_{p}^{2}$. Together with our previous observation of a divergence of $f_{c}$ near the melting temperature in Ref. 16, these results provide detailed experimental evidence for the phenomenological ordering theory of Koshelev and Vinokur. ${ }^{1}$

\section{ACKNOWLEDGMENTS}

We would like to thank A. Koshelev for stimulating discussion. This work was supported by the "Stiching voor Fundamenteel Onderzoek der Materie" (FOM) and the ESFVortex program. 
${ }^{1}$ A.E. Koshelev and V.M. Vinokur, Phys. Rev. Lett. 73, 3580 (1994).

${ }^{2}$ T. Giamarchi and P. Le Doussal, Phys. Rev. Lett. 76, 3408 (1996); P. Le Doussal and T. Giamarchi, Phys. Rev. B 57, 11356 (1998).

${ }^{3}$ L. Balents, M.C. Marchetti, and L. Radzihovsky, Phys. Rev. B 57, 7705 (1998); L. Balents and M.P.A. Fisher, Phys. Rev. Lett. 75, 4270 (1995).

${ }^{4}$ S. Scheidl and V.M. Vinokur, Phys. Rev. B 57, 13800 (1998); Phys. Rev. E 57, 2574 (1998).

${ }^{5}$ M.C. Faleski, M.C. Marchetti, and A.A. Middleton, Phys. Rev. B 54, 12427 (1996).

${ }^{6}$ K. Moon, R.T. Scalettar, and G.T. Zimanyi, Phys. Rev. Lett. 77, 2778 (1996); S. Spencer and H.J. Jensen, Phys. Rev. B 55, 8473 (1997); A.B. Kolton, D. Dominguez, and N. Grønbech-Jensen, Phys. Rev. Lett. 83, 3061 (1999).

${ }^{7}$ A.B. Kolton, D. Dominguez, and N. Gronbech-Jensen, Phys. Rev. Lett. 86, 4112 (2001).

${ }^{8}$ M. Marchevsky, J. Aarts, P.H. Kes, and M.V. Indenbom, Phys. Rev. Lett. 78, 531 (1997).

${ }^{9}$ F. Pardo, F. de la Cruz, P.L. Gammel, E. Bucher, and D.J. Bishop, Nature (London) 396, 348 (1998).

${ }^{10}$ A.M. Troyanovski, J. Aarts, and P.H. Kes, Nature (London) 399, 665 (1999).

${ }^{11}$ S. Bhattacharya and M.J. Higgins, Phys. Rev. Lett. 70, 2617 (1993).

${ }^{12}$ M.C. Hellerqvist, D. Ephron, W.R. White, M.R. Beasley, and A. Kapitulnik, Phys. Rev. Lett. 76, 4022 (1996); M.C. Hellerqvist and A. Kapitulnik, Phys. Rev. B 56, 5521 (1997).

${ }^{13}$ J.M.E. Geers, C. Attanasio, M.B.S. Hesselberth, J. Aarts, and P.H. Kes, Phys. Rev. B 63, 094511 (2001).

${ }^{14}$ Y. Paltiel, Y. Myasoedov, E. Zeldov, G. Jung, M.L. Rappaport, D.E. Feldman, M.J. Higgins, and S. Bhattacharya, Phys. Rev. B 66, 060503 (2002).

${ }^{15}$ K.E. Bassler, M. Paczuski, and E. Altshuler, Phys. Rev. B 64, 224517 (2001).

${ }^{16}$ R. Besseling, N. Kokubo, and P.H. Kes, Phys. Rev. Lett. 91, 177002 (2003).

${ }^{17}$ Y. Togawa, R. Abiru, K. Iwaya, H. Kitano, and A. Maeda, Phys. Rev. Lett. 85, 3716 (2000).

${ }^{18}$ A.T. Fiory, Phys. Rev. Lett. 27, 501 (1971); Phys. Rev. B 7, 1881 (1973); 8, 5039 (1973); J.M. Harris, N.P. Ong, R. Gagnon, and L. Taillefer, Phys. Rev. Lett. 74, 3684 (1995); Y. Togawa, H. Kitano, and A. Maeda, Physica C 378, 448 (2002).

${ }^{19}$ P. Martinoli, O. Daldini, C. Leemann, and E. Stocker, Solid State Commun. 17, 205 (1975).

${ }^{20}$ L. Van Look, E. Rosseel, M.J. Van Bael, K. Temst, V.V. Moshchalkov, and Y. Bruynseraede, Phys. Rev. B 60, R6998 (1999).

${ }^{21}$ N. Kokubo, R. Besseling, V.M. Vinokur, and P.H. Kes, Phys. Rev. Lett. 88, 247004 (2002).

${ }^{22}$ G. Grüner, Rev. Mod. Phys. 60, 1129 (1988).

${ }^{23}$ R.E. Thorne, W.G. Lyons, J.W. Lyding, J.R. Tucker, and J. Bardeen, Phys. Rev. B 35, 6360 (1987).

${ }^{24}$ Ch. Leeman, Ph. Lerch, and P. Martinoli, Physica B 126, 475 (1984); S.P. Benz, M.S. Rzchowski, M. Tinkham, and C.J. Lobb, Phys. Rev. Lett. 64, 693 (1990); K. Ravindran, L.B. Gomez, R.R. Li, S.T. Herbert, P. Lukens, Y. Jun, S. Elhamri, R.S. Newrock, and D.B. Mast, Phys. Rev. B 53, 5141 (1996).

${ }^{25}$ A. Pruymboom, P.H. Kes, E. van der Drift, and S. Radelaar, Phys.
Rev. Lett. 60, 1430 (1988); M.H. Theunissen, E. Van der Drift, and P.H. Kes, ibid. 77, 159 (1996).

${ }^{26}$ A. Schmid and W. Hauger, J. Low Temp. Phys. 11, 667 (1973).

${ }^{27}$ A.I. Larkin and Yu.N. Ovchinikov, Sov. Phys. JETP 38, 854 (1974).

${ }^{28}$ P. Martinoli, Phys. Rev. B 17, 1175 (1978).

${ }^{29}$ H. Matsukawa, J. Phys. Soc. Jpn. 56, 1507 (1987); 56, 1522 (1987).

${ }^{30}$ J. McCarten, D.A. DiCarlo, and R.E. Thorne, Phys. Rev. B 49, 10113 (1994).

${ }^{31}$ M. Tinkham, Introduction to Superconductivity (McGraw-Hill, New York, 1996).

${ }^{32}$ For convenience we use the same symbol $f_{p}$ both for the periodic and the random pinning cases. In the following it will be clear from the context which one we use.

${ }^{33}$ For sinusoidal pinning, $\Delta j\left(f \ll f_{p}\right)$ can be obtained analytically for small rf amplitude $j_{\mathrm{rf}} \ll j_{c}$, see M.J. Renne and D. Polder, Rev. Phys. Appl. 9, 25 (1974). However, both our simulations and those in Ref. 34 show that the maximum value $\Delta j_{\max }(f$ $\left.\ll f_{p}\right)$ is achieved for $j_{\mathrm{rf}} \simeq j_{c}$, for which the analytical approach fails.

${ }^{34}$ P. Russer, J. Appl. Phys. 43, 2008 (1972).

${ }^{35}$ M. Octavio, J.U. Free, S.P. Benz, R.S. Newrock, D.B. Mast, and C.J. Lobb, Phys. Rev. B 44, 4601 (1991).

${ }^{36}$ R. Besseling, R. Niggebrugge, and P.H. Kes, Phys. Rev. Lett. 82, 3144 (1999).

${ }^{37}$ R. Besseling, T. Dröse, V.M. Vinokur, and P.H. Kes, Europhys. Lett. 62, 419 (2003).

${ }^{38}$ R. Besseling, Ph. D. thesis, Leiden University, 2001.

${ }^{39}$ R. Besseling et al. (unpublished).

${ }^{40}$ E. van der Drift, S. Radelaar, A. Pruymboom, and P.H. Kes, J. Vac. Sci. Technol. B 6, 297 (1988).

${ }^{41}$ In recent measurements on "bulk" $\mathrm{NbGe}$ films where pinning originates from intrinsic disorder in the film, $\Delta I\left(I_{\mathrm{rf}}\right)$ exhibited an even closer correspondence to the relation $\Delta I\left(I_{\mathrm{rf}}\right) \propto J_{1}^{2}(z)$ at high frequency, see R. Besseling, O. Benningshof, N. Kokubo, and P.H. Kes, cond-mat/0304046 (unpublished).

${ }^{42}$ G.J.C. van Baarle, A.M. Troianovski, T. Nishizaki, P.H. Kes, and J. Aarts, Appl. Phys. Lett. 82, 1081 (2003).

${ }^{43}$ In the 1D simulations (both for ordered and disordered channels) a similar saturation of the "optimum" rf current was observed for the ML steps with $f \downarrow 0$, see also Refs. 33,34.

${ }^{44}$ P. Berghuis and P.H. Kes, Phys. Rev. B 47, 262 (1993).

${ }^{45}$ A.I. Larkin and Yu. Ovchinikov, J. Low Temp. Phys. 34, 409 (1979).

${ }^{46}$ N. Kokubo, J. Aarts, and P.H. Kes, Phys. Rev. B 64, 014507 (2001).

${ }^{47}$ In reality the row spacing $b$ away from a matching field will be in between $b_{M}$ and $b_{0}$. Therefore the true behavior of $L_{\mathrm{ML}} / L$ will lie between the dashed and the full curve in Fig. 5(c).

${ }^{48}$ A.B. Kolton, R. Exartier, L.F. Cugliandolo, D. Dominguez, and N. Grønbech-Jensen, Phys. Rev. Lett. 89, 227001 (2002).

${ }^{49}$ V.M. Vinokur, P.H. Kes, and A.E. Koshelev, Physica C 168, 29 (1990).

${ }^{50}$ A.B. Pippard, Philos. Mag. 19, 217 (1969).

${ }^{51}$ N. Kokubo, R. Besseling, and P.H. Kes, cond-mat/0308372 (unpublished).

${ }^{52}$ We note that $\delta V_{1,1}$ is much too large to be attributed to a spread in $j_{c}$ from channel to channel, which was further confirmed by 
measurements on only 30 channels.

${ }^{53}$ For CDW's, (broadened) narrow band noise is generally present in the dc case. Additional rf driving leads to further synchronization at mode locking, as shown in S. Bhattacharya, J.P. Stokes,
M.J. Higgins, and R.A. Klemm, Phys. Rev. Lett. 59, 1849 (1987). This is consistent with a diverging velocity correlation length at ML, which was theoretically predicted by Matsukawa (Ref. 29). 